\title{
Scheduling design of Jakarta-Cikampek II elevated toll road project (P.186 - P.187)
}

\author{
Abdul Khalim ${ }^{1 *}$, Harun Usman Ghifarsyam ${ }^{2}$, Nikko Rozy ${ }^{3}$, Faqih Ma'arif ${ }^{4}$ \\ ${ }^{1}$ Department of Civil Engineering, Beijing Jiaotong University, Beijing, China \\ ${ }^{2}$ Department of Computer Science, Beijing Jiaotong University, Beijing, China \\ ${ }^{3}$ Swadaya Gunungjati University, Cirebon, Indonesia \\ ${ }^{4}$ Department of Civil Engineering, Beihang Unviersity, Beijing, China \\ *E-mail: abdoel553@outlook.com \\ * corresponding author
}

ABSTRACT

ARTICLE INFO

The key to achieving effective and efficient development goals is by increasing the quality of construction management by collecting up-to-date data about the project's resources, mainly about productivity. In this research space, productivity will be discussed, including human resources and their management. The case study is the project of Jakarta-Cikampek II Elevated Toll Road. This project was a developing project to improve the capacity of the existing Jakarta-Cikampek toll road. This elevated structure will be built right in the area (median and side edge) of the existing toll road, making it interesting to discuss how the construction is carried out. Scheduling analysis in this study was carried out at points P.186 to P.187 of the construction project. The analysis includes identifying the productivity index of labor resources and equipment that has a major role in shaping the scheduling concept. The concept will be performed by determining a network diagram that will use the Critical Path Method (CPM) rule. The data used in this analysis are construction drawing, scurve plan, worker data, weekly job plan, work method, and some interviews with worker and field project manager. These results obtained the large productivity of workers and equipment, and the duration required to complete the construction starting prom point P.186 to P.187 is 248 working days.

This is an open-access article under the $\underline{\mathrm{CC}-\mathrm{BY}-\mathrm{SA}}$ license

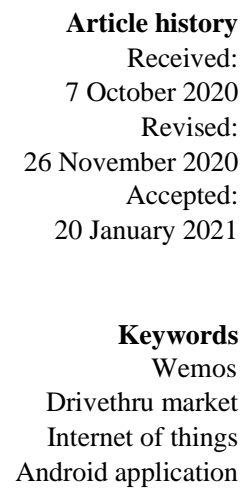

Article history

Received:

7 October 2020

Revised:

26 November 2020

Accepted:

20 January 2021

Keywords

Wemos

Drivethru market

Internet of things

Android application

\section{Introduction}

The Project of Jakarta-Cikampek II Elevated Toll Road will be built right in the median area and on the side of the existing toll road. This project's duration will greatly affect the conduciveness of traffic, especially in the Jakarta-Cikampek toll road area. It needs an effective and efficient construction management plan to be done and completed properly.

Management is coordinating work activities so that the work is completed efficiently and effectively and through other researchers [1], [2]. The research aims to plan a construction 
Journal of Engineering and Applied Technology

Vol. 1, No. 2, August 2020, pp. 51-65

management concept within the scope of effective and efficient scheduling to minimize disruption to the currently active toll road. The research method must include productivity analysis of human resources and tools. The data must be as accurate as possible because it will be used to determine the work duration and compile it into a network diagram using the CPM (Critical Path Method) method.

In this study, the information on workers in the form of numbers and their productivity during working at a certain time will determine the productivity index of a worker in a particular job. The tools used and which play an important role in the completion of a job will also be identified for their number, specifications, and productivity. The productivity will eventually be used to determine how long it takes for tools and workers to complete their work. The optimal duration will be obtained, which will be used to compile a network diagram and analyze it using the Critical Path Method (CPM).

\section{Method}

The productivity of equipment are as follows:

\subsection{Excavator}

(1) Bucket Capacity (m3) V

(2) Bucket Factor

$\mathrm{Fb}$

(3) Equipment efficiency factor

$\mathrm{Fa}$

(4) Conversion factor, Depth $<40 \%$

Fv

(5) Time cycle

Tc

(6) Digging (minute)

$\mathrm{T} 1$

(7) Swing and Dump (minute)

$\mathrm{T} 2$

(8) Setting Position (minute)

T3

(9) Disruption (minute)

$\mathrm{T} 4$

(10) Time cycle (minute) $(\mathrm{T} 1+\mathrm{T} 2+\mathrm{T} 3+\mathrm{T} 4) \times \mathrm{Fv})$

Tc

(11) Prod./Hour (m3/hour)

Q Excavator

Table 1. The bucket fill factor of an excavator $(\mathrm{Fa})$ from Regulation of the Minister of Public Works No. 282016 [3]

\begin{tabular}{llc}
\hline \multicolumn{1}{c}{ Operating condition } & \multicolumn{1}{c}{ Field condition } & Bucket factor $(\mathbf{F b})$ \\
\hline Easy & Common ground, clayey soil, soft soil & $1.1-1.2$ \\
Average & Sandy soil, dry soil & $1.0-1.1$ \\
Rather difficult & Sandy soil with gravel & $1.0-0.9$ \\
Difficult & Blasted rock & $0.9-0.8$ \\
\hline
\end{tabular}

Table 2. The efficiency factor of an excavator ( $\mathrm{Fa})$ from Regulation of the Minister of Public Works No. 282016

\begin{tabular}{lc}
\hline \multicolumn{1}{c}{ Operating condition } & Efficiency Factor $(\mathbf{F a})$ \\
\hline Good & $1.1-1.2$ \\
Average & $1.0-1.1$ \\
Rather poor & $1.0-0.9$ \\
Poor & $0.9-0.8$ \\
\hline
\end{tabular}


Journal of Engineering and Applied Technology

Vol. 1, No. 2, August 2020, pp. 51-65

Table 3. Conversion factor (Fv)

from Regulation of the Minister of Public Works No. 282016 [3]

\begin{tabular}{|c|c|c|c|c|}
\hline \multirow{2}{*}{$\begin{array}{c}\text { Digging } \\
\text { Condition } \\
\left(\frac{\text { Digging condition }}{\text { digging depth }}\right)\end{array}$} & \multicolumn{4}{|c|}{ Dumping condition } \\
\hline & 空 & 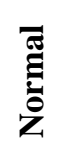 & & 氖 \\
\hline$<40 \%$ & 0.7 & 0.9 & 1.1 & 1.4 \\
\hline$(40-75) \%$ & 0.8 & 1.0 & 1.3 & 1.6 \\
\hline$>75 \%$ & 0.9 & 1.1 & 1.5 & 1.8 \\
\hline
\end{tabular}

$$
\text { Qexcavator }=\frac{\mathrm{V} \times \mathrm{Fb} \times \mathrm{Fa} \times 60}{\mathrm{Tc}}
$$

\subsection{Wheel Loader}

(1) Average speed $(\mathrm{km} / \mathrm{h})$

V

(2) Number of passes (passes)

n

(3) Efficiency factor (good $=0.83) \quad \mathrm{Fe}$

(4) Compaction width effectivity be

(5) Compaction thickness (m) t

(6) Productivity/hour $(\mathrm{m} 3 / \mathrm{h}) \quad Q_{\text {vibraroller }}$

$$
\text { Qvibraroller }=\frac{(\text { be } \mathrm{xv} \times 1000) \times \mathrm{t} \times \mathrm{Fe}}{\mathrm{n}}
$$

\subsection{Dump Truck}

(1) Bed Capacity (m3)

(2) Loaded average speed $(\mathrm{km} / \mathrm{h})$

\section{V}

(3) Empty average speed $(\mathrm{km} / \mathrm{h})$

v1

v2

(4) Time cycle

Tc

(5) Loading (V/ Q1) x 60

T1

(6) Loaded travel time (L/ V1) x 60

$\mathrm{T} 2$

(7) Empty travel time (L / V2) x 60

T3

(8) Time cycle $\left(\sum \mathrm{T}\right)$

Tc

(9) Productivity/hour (m3/h)

Qdump truck

Table 4. Job Efficiency (Fe)

from Regulation of the Minister of Public Works No. 282016 [3]

\begin{tabular}{lc}
\hline \multicolumn{1}{c}{ Work condition } & Job efficiency $(\mathbf{F e})$ \\
\hline Good & 0.83 \\
Average & 0.80 \\
Rather poor & 0.75 \\
poor & 0.70 \\
\hline
\end{tabular}


Journal of Engineering and Applied Technology

Vol. 1, No. 2, August 2020, pp. 51-65

Table 5. Average speed (Fa)

\begin{tabular}{ccc}
\hline Field condition & Loading condition & Speed/ v (km/h) \\
\hline Flat & Loaded & 40 \\
& Unloaded & 60 \\
Uphill & Loaded & 20 \\
& Unloaded & 40 \\
\multirow{2}{*}{ Downhill } & Loaded & 20 \\
& Unloaded & 40 \\
\hline
\end{tabular}

$$
\text { Qdumptruck }=\frac{\mathrm{V} \times \mathrm{Fe} \times 60}{\mathrm{D} \times \mathrm{Fk} \times \mathrm{Tc}}
$$

\subsection{Flatbed Truck}

(1) Bed Capacity (unit of RCP) V

(2) Efficiency factor $\mathrm{Fe}$

(3) Loaded average speed v1

(4) Empty average speed v2

(5) Time Cycle $\left(\sum \mathrm{T}_{1,2,3,4}\right) \quad \mathrm{Tc}$

(6) Loaded travel time (L/v1) x $60 \quad \mathrm{~T} 1$

(7) Empty travel time (L/v2) x $60 \quad$ T2

(8) Loading T3

(9) RCP erection $\quad \mathrm{T} 4$

(10) Productivity/hour (m'/hour) Qflatbed

\subsection{Drill Machine SR60}

$$
\text { Qflatbed }=\frac{\mathrm{V} \times \mathrm{Fe} \times 60}{\mathrm{Tc}}
$$

(1) Production Capacity (m'/hour) Q1

(2) Production Capacity (m3/hour) Q1'

$$
\mathrm{Q} 1^{\prime}=0.25 \pi \times \mathrm{D} 2 \times \mathrm{Q} 1
$$

\subsection{Water Tank Truck}

(1) Bucket Capacity (m3) V

(2) Bucket Factor $\mathrm{Fb}$

(3) Efficiency factor (good $=0.83$ ) $\quad \mathrm{Fe}$

(4) Conversion factor, Depth $<40 \% \quad \mathrm{Fv}$

(5) Time cycle $\quad \mathrm{Tc}$

(6) Digging (V / Q1') x 60(minute) $\mathrm{T} 1$

(7) Swing and Dump (minute) T2

(8) Disruption (minute) $\mathrm{T} 4$

(9) Time cycle $(\mathrm{T} 1+\mathrm{T} 2+\mathrm{T} 3+\mathrm{T} 4) \mathrm{x} \mathrm{Fv}) \quad \mathrm{Tc}$

(10) Prod./Hour (m3/hour) Qwatertruck 
Journal of Engineering and Applied Technology

Vol. 1, No. 2, August 2020, pp. 51-65

$$
\text { Qwatertank }=\frac{\mathrm{V} \times \mathrm{Fb} \times \mathrm{Fe} \times 60}{\mathrm{Tc}}
$$

(11) Capacity (m3)

(12) Efficiency factor ( good $=0.83$ )

(13) Loaded average speed

(14) Empty average speed

(15) Time cycle

(16) Filling

(17) Transport (L: V1) x 60

(18) Reverse (L: V2) x 60

(19) Setting, idling, pouring

(20) Productivity/hour (m3/hour)
V

$\mathrm{Fe}$

V1

$\mathrm{V} 2$

Tc

T1

$\mathrm{T} 2$

$\mathrm{T} 3$

$\mathrm{T} 4$

Q truckmixer

$$
\text { Qtruckmixer }=\frac{\mathrm{V} \times \mathrm{Fe} \times 60}{\mathrm{Tc}}
$$

\subsection{Crawler Crane}

(1) Lifting Capacity (ton)

\section{V}

(2) Efficiency factor (good $=0,83) \mathrm{Fe}$

(3) Time cycle

Tc3

(4) Binding

T1

(5) Lifting

$\mathrm{T} 2$

(6) Swing

T3

(7) Setting, Holding \& Uninstall Pipe

T4

(8) Productivity/hour (ton/hour)

$\mathrm{Q}_{\text {tremiepipe }}$

$$
\text { Qcrawlercrane }=\frac{\mathrm{V} \times \mathrm{Fe} \times 60}{\mathrm{Tc}}
$$

\subsection{Wheel Loader}
(1) Bucket Capacity
V
(2) Bucket factor
$\mathrm{Fb}$
(3) Equipment efficiency factor
$\mathrm{Fe}$
(4) Forward average speed
$\mathrm{Vf}$
(5) Reverse average speed
$\mathrm{Vr}$
(6) Time cycle (mnt)
Tc
(7) Loading to Bin (mnt)
T1
(8) Reverse to Stock Bin (mnt)
$\mathrm{T} 2$
(9) Disruption (mnt)
T3
(10) Productivity/ hour (m2/h)
Q1 
Journal of Engineering and Applied Technology

Vol. 1, No. 2, August 2020, pp. 51-65

Table 6. Bucket factor of wheel loader $(\mathrm{Fa})$

from Regulation of the Minister of Public Works No. 282016 [3]

\begin{tabular}{lc}
\hline Pouring condition & Bucket factor \\
\hline Easy & $1.0-1.1$ \\
Average & $0.85-0.95$ \\
Rather difficult & $0.80-0.85$ \\
Difficult & $0.75-0.80$ \\
\hline
\end{tabular}

$$
\text { Qwheelloader }=\frac{\mathrm{V} \times \mathrm{Fb} \times \mathrm{Fe} \times 60 \times \mathrm{Ws}}{\mathrm{Tc}}
$$

\subsection{Pneumatic Tire Roller}

(1) Average speed $(\mathrm{km} / \mathrm{hr})$

$\mathrm{V}$

(2) Effective width compaction (m) b

(3) Number of passes (passes) n

(4) Number of line $\mathrm{N}$

(5) Equipment efficiency factor $\quad \mathrm{Fe}$

(6) Overlap width (m) bo

(7) Productivity/hour (ton/hour) Q Qtireroller

$$
\text { Qtireroller }=\frac{(\mathrm{V} \times 1000) \times(\mathrm{N}(\mathrm{b}-\mathrm{bo})+\mathrm{bo}) \mathrm{t} \times \mathrm{Fe} \times \mathrm{D} 1}{\mathrm{n}}
$$

\subsection{Productivity of Manpower}

Manpower productivity is identified by comparing the output and the time required for a job [4].

$$
\text { Productivity/day: } \mathrm{Q}_{\mathrm{t}}=\mathrm{T}_{\mathrm{w}} \times \mathrm{Q}_{1}
$$

(1) Time working/day

Tw

(2) Digging Productivity/Day

Qt

$$
\mathrm{Qt}=\mathrm{Tw} \times \mathrm{Q} 1
$$

Manpower:

(1) Worker

(2) Foreman

W

$$
\text { f }
$$

Manpower Coefficient/M3:

(1) Worker $=(\mathrm{Tw} \times \mathrm{w}) / \mathrm{Qt}$

(2) Foreman $=($ Tw x f) $/ \mathrm{Qt}$

The management function approach shows apparent effectiveness or obligation when coordinating others efficiently and effectively - the four essential functions of management [1]: planning, 
Journal of Engineering and Applied Technology

Vol. 1, No. 2, August 2020, pp. 51-65

organizing, lead, controlling. In the analysis of the network, there are two concepts, namely events and activity.

(1) Events : is the beginning or end of an activity.

(2) Activity : is a job or task where the completion requires a certain period, costs, and facilities.

To design a project network, three important elements must be known [5], [6].

(1) Inventory of activity

(2) The process of inventory of these activities is the breakdown of a project into several major components of the project

(3) Dependency Logic

(4) Solving the project into work packages should consider the sequence of work to be performed

(5) Estimated Time

(6) This time estimate is the period required to complete each activity

Several factors determine the length of activity, job volume, labor, weather, project location, time estimating procedure. The terms used in the network diagram are as follows [7], [8]:

(1) Earliest Start Time (ES)is the earliest time an activity can start, taking into account the expected activity time and the sequence requirements of the exhortation.

(2) Latest Start Time (LS) is the slowest time to be able to start an activity without delaying the entire project.

(3) Earliest Finish Time (EF) is the earliest time an activity can be completed

(4) Latest Finish Time (LF) at the latest to be able to complete an activity without delaying the completion of the overall project.

(5) Duration (D) is the period of activity.

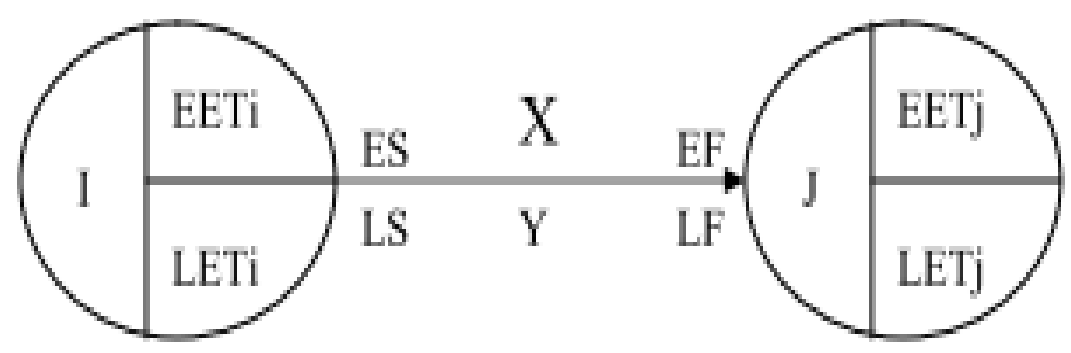

Fig. 1. Activity on Arrow (AOA)

Activity on arrow or often referred to as CPM (Critical Path Method) consists of:
(1) $\mathrm{i}, \mathrm{j}=$ Case number
(2) $\mathrm{X}=$ Activity name
(3) EET = Earliest Event Time
(4) LET = Latest Event Time
(5) $\mathrm{Y}=$ Activity Duration
(6) $\mathrm{ES} \quad=$ Earliest Start Time
(7) $\mathrm{EF} \quad=$ Earliest Finish Time
(8) $\mathrm{LS}=$ Latest Start Time 
Journal of Engineering and Applied Technology

Vol. 1, No. 2, August 2020, pp. 51-65

(9) $\mathrm{LF} \quad=$ Latest Finish Time

Calculation:

Forward pass:

(1) Early Start = Maximum (or Highest) EF value from immediate predecessor(s)

(2) Early Finish $=$ ES + Duration

Backward pass:

(1) Late Start $=\mathrm{LF}-$ Duration

(2) Late Finish = Minimum (or Lowest) LS value from immediate Successor(s)

Float:

(1) Total Float $=\mathrm{LS}-\mathrm{ES}$ (it is also calculated by LF - EF)

(2) Free Float $=$ Lowest ES of successors - EF

Critical Path method has the following characteristics:

(1) Network A network diagram is created using arrows to illustrate the activities, and the node describes an event. At the beginning of the arrow, the node is determined as I-Node, while at the end of the arrow is specified as J-Node.

(2) Use advanced calculations to obtain the earliest start time (EETi) on the earliest I-Node and start time (EET) on the J-Node of all activities by taking its maximum value. Here it is understood that the earliest time of event occurs $=0$. The calculation is EETj $=E E T i+$ duration $X$.

(3) Use the countdown to obtain the slowest completion time (LETi) on the I-Node and the slowest completion time (LETj) on the J-Node of all activities by taking the minimum value. The calculation is $\mathrm{LET} i=\mathrm{LET} \mathrm{j}-\mathrm{X}$ duration. $\mathrm{LETi}=\mathrm{LET} \mathrm{j}-\mathrm{X}$ duration .

(4) Between two events, there is a CPM (Critical Path Method), where a deterministic approach uses only one type of duration on its activities. Total Float $(\mathrm{TF})=0$.

(5) Float: the tolerance limit for the delay in an activity that can be used for time optimization and resource allocation.

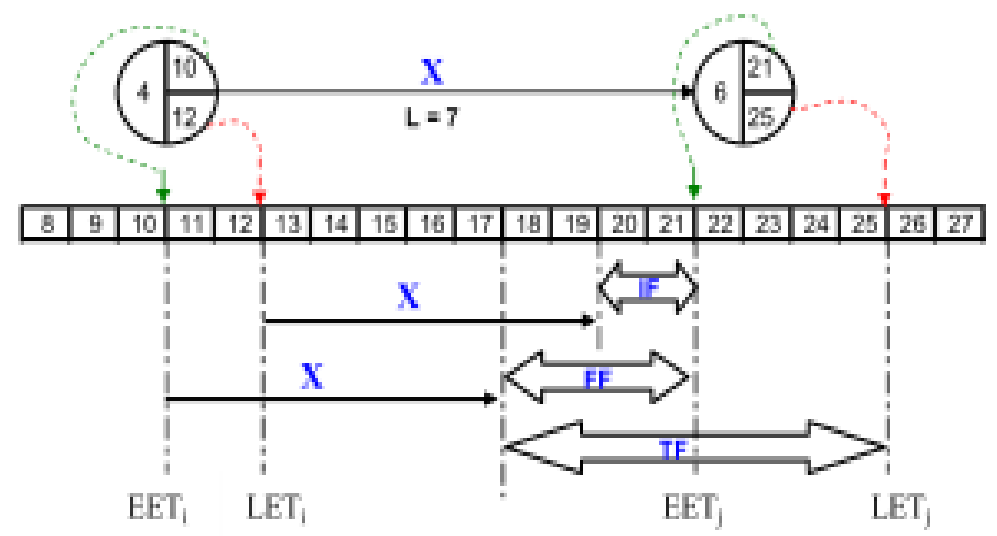

Fig. 2. Float variation of an activity 
Journal of Engineering and Applied Technology

Vol. 1, No. 2, August 2020, pp. 51-65

\section{Results and Discussion}

Productivity identification of tools that had a vital role in developing construction is presented in Table 7. Labor productivity has differences in each type of work [9]-[11] as Fig. 3 shows different labor productivity in several jobs based on the duration of the number of workers according to conditions in the field [12].

Table 7. Productivity value of main tools

\begin{tabular}{lcc}
\hline \multicolumn{1}{c}{ Equipment } & Job section & Prod. \\
\hline Excavator Komatsu PC128US-2 & & $59.94 \mathrm{~m} 3 / \mathrm{hr}$ \\
Dump Truck Hino FM 260 JD & Work Platform and compaction & $16.48 \mathrm{~m} 3 / \mathrm{hr}$ \\
Vibrator Roller CatCB54B & & $136 \mathrm{~m} 3 / \mathrm{hr}$ \\
Excavator Komatsu PC128US-2 & & $20.54 \mathrm{~m} 3 / \mathrm{hr}$ \\
Dump Truck Hino FM 260 JD & Drainage & $7.53 \mathrm{~m} 3 / \mathrm{hr}$ \\
Flatbed Truck Tata LPS 4018 TC EX & & $7.45 \mathrm{~m} / \mathrm{hr}$ \\
Drill Machine SR60 & & $11.03 \mathrm{~m} / \mathrm{hr}$ \\
Watertank Truck Henghe HHR5160GSS4EQ & Drilling Bored Pile & $9.91 \mathrm{~m} 3 / \mathrm{hr}$ \\
Dump Truck Hino FM 260 JD & & $1.28 \mathrm{~m} 3 / \mathrm{hr}$ \\
Service Crane (Crawler) & & \\
Concrete Mixer Hino FM 260 JM & Bored Pile Concrete Casting & $22.20 \mathrm{ton} / \mathrm{hr}$ \\
Crawler crane & Driving Steel Sheet Pile & $7 \mathrm{sheet} / \mathrm{hr}$ \\
Excavator Vibro mounted YZM & Pile cap & $29.88 \mathrm{~m} 3 / \mathrm{hr}$ \\
Excavator Kobelco SK-200 & & $9.03 \mathrm{~m} 3 / \mathrm{hr}$ \\
Dump Truck Hino FM 260 JD & Reinforcement & $199.20 \mathrm{~kg} / \mathrm{hr}$ \\
Bar Cutter \& Bender & & $420 \mathrm{~kg} / \mathrm{hr}$ \\
Bar Roller & Concrete Casting, Pile Cap, Pier, & $6.23 \mathrm{~m} 3 / \mathrm{hr}$ \\
Concrete Mixer Hino FM 260 JM & Slab & $21.86 \mathrm{~m} 3 / \mathrm{hr}$ \\
Concrete Pump & & $3.00 \mathrm{~m} 3 / \mathrm{hr}$ \\
Concrete Vibrator REDFOX & & $47.52 \mathrm{~m} 2 / \mathrm{hr}$ \\
Wheel Loader CDM816 & & $3.37 \mathrm{~m} 3 / \mathrm{hr}$ \\
Dump Truck Hino FM 260 JD & & $48.11 \mathrm{ton} / \mathrm{hr}$ \\
Asphalt finisher Sumitomo HB45W & & \\
Tandem roller Sakai SW350 & & $21.99 \mathrm{ton} / \mathrm{hr}$ \\
Pneumatic Tire Roller Sakai TS7409 & & $51.26 \mathrm{ton} / \mathrm{hr}$ \\
Thermoplastic Machine Jili Jlcrj & & $9.34 \mathrm{~m} 2 / \mathrm{hr}$ \\
Air Compressor & & $8.305 \mathrm{~m} 2 / \mathrm{hr}$ \\
\hline
\end{tabular}


Journal of Engineering and Applied Technology

Vol. 1, No. 2, August 2020, pp. 51-65

\begin{tabular}{|c|c|c|c|c|c|c|}
\hline & & Rebar in & allation & & & \\
\hline 1 & Rebar installation & & & & & \\
\hline & Volume & & & $\mathrm{v}$ & 18157,9 & $9 \mathrm{Kg}$ \\
\hline & duration plan (Specification & & & D & 5 & 5 Day \\
\hline & Labor & & & w & 11 & \\
\hline & - Labor & & & w & & 6 Man \\
\hline & - Skilled labor & & & tw & & 2 Man \\
\hline & - Skilled labor & & & tw & & 1 Man \\
\hline & - Foreman & & & $\mathrm{m}$ & & 1 Man \\
\hline & Productivity Kg/Day & $=\frac{V}{D}$ & & $\mathrm{Q}$ & 3631,59 & $9 \mathrm{Kg} / \mathrm{Day}$ \\
\hline & Coefficient of Manpower & & & & & \\
\hline & - Labor & 6 Man & $=6: Q$ & & 0,00165 & 5 MD \\
\hline & - Skilled labor & 2 Man & $=2: Q$ & & 0,00055 & $5 \mathrm{MD}$ \\
\hline & - Skilled labor & 1 Man & $=1: Q$ & & 0,00028 & $8 \mathrm{MD}$ \\
\hline & - Foreman & $1 \mathrm{Man}$ & $=1: Q$ & & 0,00028 & $8 \mathrm{MD}$ \\
\hline 1 & Rebar Pier installation & & & & & \\
\hline & Volume (Specification) & & & $\mathrm{v}$ & 4815,27 & $7 \mathrm{Kg}$ \\
\hline & duration plan & & & $\mathrm{D}$ & 1 & 1 Day \\
\hline & Labor & & & w & 6 & 6 \\
\hline & - Labor & & & w & & 2 Man \\
\hline & - Skilled labor & & & tw & 2 & 2 Man \\
\hline & - Skilled labor & & & tw & & 1 Man \\
\hline & - Foreman & & & $\mathrm{m}$ & & 1 Man \\
\hline & Productivity Kg/Day & $=\frac{\mathrm{V}}{\mathrm{D}}$ & & $Q$ & 4815,27 & $7 \mathrm{Kg} /$ Day \\
\hline & Coefficient of Manpower & & & & & \\
\hline & - Labor & 2 Man & $=2: Q$ & & 0,00042 & $2 \mathrm{MD}$ \\
\hline & - Skilled labor & 2 Man & $=2: Q$ & & 0,00042 & $2 \mathrm{MD}$ \\
\hline & - Skilled labor & $1 \mathrm{Man}$ & $=1: Q$ & & 0,00021 & $1 \mathrm{MD}$ \\
\hline & - Foreman & 1 Man & $=1: Q$ & & 0,00021 & $1 \mathrm{MD}$ \\
\hline 1 & Rebar Pier Head installation & & & & & \\
\hline & Volume (Specification) & & & $\mathrm{v}$ & 14936,7 & $7 \mathrm{Kg}$ \\
\hline & duration plan & & & D & & 7 Day \\
\hline & Labor & & & w & 10 & \\
\hline & - Labor & & & w & & 6 Man \\
\hline & - Skilled labor & & & tw & 2 & 2 Man \\
\hline & - Skilled labor & & & tw & 1 & 1 Man \\
\hline & - Foreman & & & $\mathrm{m}$ & & 1 Man \\
\hline & Productivity Kg/Day & $=\mathrm{V}$ & & Q & 2133,81 & $1 \mathrm{~kg} / \mathrm{Day}$ \\
\hline & & $\mathrm{D}$ & & & & \\
\hline & Coefficient of Manpower & & & & & \\
\hline & - Labor & 6 Man & $=6: Q$ & & 0,00281 & $1 \mathrm{MD}$ \\
\hline & - Skilled labor & 2 Man & $=2: Q$ & & 0,00094 & $4 \mathrm{MD}$ \\
\hline & - Skilled labor & 1 Man & $=1: Q$ & & 0,00094 & $4 \mathrm{MD}$ \\
\hline & - Foreman & 1 Man & $=1: Q$ & & 0,00047 & $7 \mathrm{MD}$ \\
\hline 1 & Rebar Slab \& Barrier installa & ation & & & & \\
\hline & Volume (Specification) & & & $\mathrm{v}$ & 196941 & $1 \mathrm{Kg}$ \\
\hline & duration plan & & & D & 20 & o Day \\
\hline & Labor & & & w & 22 & \\
\hline & - Labor & & & w & 14 & 4 Man \\
\hline & - Skilled labor & & & tw & 6 & 6 Man \\
\hline & - Skilled labor & & & $\mathrm{m}$ & 1 & 1 Man \\
\hline & - Foreman & & & $\mathrm{m}$ & & 1 Man \\
\hline & Productivity Kg/Day & $=\mathrm{V}$ & & $\mathrm{Q}$ & 9847,05 & 5 Kg/Day \\
\hline & & $\mathrm{D}$ & & & & \\
\hline & Coefficient of Manpower & & & & & \\
\hline & - Labor & 14 Man & $=14: Q$ & & 0,00142 & $2 \mathrm{MD}$ \\
\hline & - Skilled labor & 6 Man & $=6: Q$ & & 0,00061 & $1 \mathrm{MD}$ \\
\hline & - Skilled labor & 1 Man & $=1: Q$ & & 0,0001 & $1 \mathrm{MD}$ \\
\hline & - Foreman & $1 \mathrm{Man}$ & $=1: Q$ & & 0,0001 & $1 \mathrm{MD}$ \\
\hline & & Pile De & lition & & & \\
\hline$\overline{1}$ & Pile demolition & & & & & \\
\hline & Volume (Specification) & & & $v$ & & 8 Point \\
\hline & duration plan & & & D & & 3 Day \\
\hline & Labor & & & w & & $8 \mathrm{Man}$ \\
\hline & $\begin{array}{l}\text { - Labor } \\
\text { - Tukang Batu }\end{array}$ & & & $w_{t w}^{w}$ & & $\begin{array}{l}4 \text { Man } \\
2 \text { Man }\end{array}$ \\
\hline & $\begin{array}{l}\text { - Tukang Batu } \\
\text { - Tukang Batu }\end{array}$ & & & ${ }_{\mathrm{m}}^{\mathrm{tw}}$ & & 1 Man \\
\hline & - Foreman & & & $\mathrm{m}$ & & 1 Man \\
\hline & Productivity Kg/Day & $=v$ & & $\mathrm{a}$ & 2,667 & 57 Point/Day \\
\hline & Coefficient of Manpower & & & & & \\
\hline & - Labor & 4 Man & $=14: Q$ & & & $5 \mathrm{MD}$ \\
\hline & - Tukang Batu & $2 \mathrm{Man}$ & $=6: a$ & & & $15 \mathrm{MD}$ \\
\hline & $\begin{array}{l}\text { - Tukang Batu } \\
\text { - Foreman }\end{array}$ & 1 Man & $\begin{array}{l}=1: Q \\
=1: Q\end{array}$ & & 0,375 & $15 \mathrm{MD}$ \\
\hline
\end{tabular}

Fig. 3. The productivity of each labor/workers 
Journal of Engineering and Applied Technology

Vol. 1, No. 2, August 2020, pp. 51-65

\subsection{Network Diagram Using Critical Path Method (CPM)}

Table 8. CPM deterministic and critical path identification

\begin{tabular}{|c|c|c|c|c|c|c|c|c|c|c|}
\hline No & Description & Node & $\begin{array}{l}\text { Immediate } \\
\text { Predecessor }\end{array}$ & Duration & $\begin{array}{c}\text { Critical } \\
\text { Path } \\
\end{array}$ & $\mathbf{E S}$ & $\mathbf{L S}$ & $\mathbf{E F}$ & $\mathbf{L F}$ & $\begin{array}{l}\text { Slack } \\
\text { Time } \\
\end{array}$ \\
\hline \multirow[t]{3}{*}{1} & General Work & & - & & & & & & & \\
\hline & Traffic Protection and Treatment & & & & & & & & & \\
\hline & Mob and Demod & & & & & & & & & \\
\hline \multirow[t]{3}{*}{2} & Preliminary Work & & - & & & & & & & \\
\hline & Measurement And Blowplank & A & - & 2 & Yes & 0 & 0 & 2 & 2 & 0 \\
\hline & Site Office & $\mathrm{B}$ & A & 18 & Yes & 2 & 2 & 20 & 20 & 0 \\
\hline \multirow[t]{4}{*}{3} & Earthworks & & & & & & & & & 0 \\
\hline & $\begin{array}{l}\text { Site Clearing, Stripping \& } \\
\text { Grubbing }\end{array}$ & $\mathrm{C}$ & A & 9 & No & 2 & 11 & 11 & 20 & 18 \\
\hline & Land Filling \& Compaction & $\mathrm{D}$ & $\mathrm{B}, \mathrm{C}$ & 1 & Yes & 20 & 20 & 21 & 21 & 0 \\
\hline & Excavation $0-2 \mathrm{M}$ & $\mathrm{E}$ & A & 2 & No & 2 & 4 & 19 & 21 & 4 \\
\hline \multicolumn{11}{|c|}{ Substructure } \\
\hline \multirow[t]{5}{*}{4} & Bored Pile Foundation & & & & & & & & & \\
\hline & Drilling & $\mathrm{F}$ & $\mathrm{D}, \mathrm{E}$ & 10 & Yes & 21 & 21 & 31 & 31 & 0 \\
\hline & Reinforcement & $\mathrm{G}$ & $\mathrm{D}, \mathrm{E}$ & 12 & No & 21 & 33 & 25 & 37 & 24 \\
\hline & Material Removing & $\mathrm{H}$ & $\mathrm{F}$ & 10 & Yes & 31 & 31 & 41 & 41 & 0 \\
\hline & Concrete Structure & I & $\mathrm{F}, \mathrm{G}$ & 4 & No & 33 & 37 & 37 & 41 & 8 \\
\hline \multirow[t]{8}{*}{5} & Pile Cap Work & & & & & & & & & 0 \\
\hline & Ssp Driving & $\mathrm{J}$ & $\mathrm{H}, \mathrm{I}$ & 4 & Yes & 41 & 41 & 45 & 45 & 0 \\
\hline & Footing Excavation & $\mathrm{K}$ & $\mathrm{J}$ & 4 & Yes & 45 & 45 & 49 & 49 & 0 \\
\hline & Pile Demolition & $\mathrm{L}$ & $\mathrm{K}$ & 6 & No & 49 & 63 & 55 & 69 & 28 \\
\hline & Reinforcement Work & $\mathrm{M}$ & $\mathrm{K}$ & 20 & Yes & 49 & 49 & 69 & 69 & 0 \\
\hline & Formwork Installation & $\mathrm{N}$ & $\mathrm{L}, \mathrm{M}$ & 2 & Yes & 69 & 69 & 71 & 71 & 0 \\
\hline & Concrete Cast + Waiting Time & $\mathrm{O}$ & $\mathrm{N}$ & 6 & Yes & 71 & 71 & 77 & 77 & 0 \\
\hline & Curing & $\mathrm{P}$ & $\mathrm{O}$ & 14 & Yes & 77 & 77 & 91 & 91 & 0 \\
\hline \multirow[t]{6}{*}{6} & Pier & & & & & & & & & 0 \\
\hline & Reinforcement Work & Q & $\mathrm{O}$ & 10 & No & 77 & 87 & 87 & 97 & 20 \\
\hline & $\begin{array}{l}\text { Scaffolding + Climbing Platform } \\
\text { Installation }\end{array}$ & $\mathrm{R}$ & $\mathrm{P}$ & 6 & Yes & 91 & 91 & 97 & 97 & 0 \\
\hline & Formwork Installation & $\mathrm{S}$ & $\mathrm{Q}, \mathrm{R}$ & 6 & Yes & 97 & 97 & 103 & 103 & 0 \\
\hline & Concrete Cast + Waiting Time & $\mathrm{T}$ & S & 6 & Yes & 102 & 103 & 109 & 109 & 0 \\
\hline & Curing & $\mathrm{U}$ & $\mathrm{T}$ & 14 & Yes & 109 & 109 & 123 & 123 & 0 \\
\hline \multirow[t]{10}{*}{7} & Pier Head & & & & & & & & & 0 \\
\hline & Shoring Installation & $\mathrm{V}$ & $\mathrm{U}$ & 2 & No & 123 & 123 & 125 & 125 & 0 \\
\hline & Formwork Installation & W & V & 10 & No & 125 & 125 & 135 & 135 & 0 \\
\hline & Sosrobahu Tool Installation & $\mathrm{X}$ & $\mathrm{W}$ & 10 & No & 135 & 135 & 145 & 145 & 0 \\
\hline & Reinforcement Work & $\mathrm{Y}$ & U & 22 & Yes & 123 & 123 & 145 & 145 & 0 \\
\hline & Concrete Cast+ Waiting Time & $\mathrm{Z}$ & $\mathrm{W}, \mathrm{X}, \mathrm{Y}$ & 6 & Yes & 145 & 145 & 151 & 151 & 0 \\
\hline & Curing & AA & $\mathrm{Z}$ & 14 & Yes & 151 & 151 & 165 & 165 & 0 \\
\hline & Erection + Stressing Tendon & $\mathrm{AC}$ & AA & 2 & Yes & 165 & 165 & 167 & 167 & 0 \\
\hline & $\begin{array}{l}\text { Pedestal + Temporary Bearing } \\
\text { Installation }\end{array}$ & $\mathrm{AD}$ & AA & 2 & No & 165 & 167 & 165 & 167 & 4 \\
\hline & $\begin{array}{l}\text { Uninstalling Formwork + } \\
\text { Shoring }\end{array}$ & $\mathrm{AB}$ & $\mathrm{AC}, \mathrm{AD}$ & 7 & Yes & 167 & 167 & 174 & 174 & 0 \\
\hline \multicolumn{11}{|c|}{ Superstructure } \\
\hline \multirow[t]{4}{*}{8} & Steel Box Girder & & & & & & & & & \\
\hline & Sbg Mobilization & $\mathrm{AE}$ & $\mathrm{AB}$ & 1 & No & 174 & 174 & 175 & 175 & 0 \\
\hline & Erection & $\mathrm{AF}$ & $\mathrm{AB}$ & 1 & Yes & 174 & 174 & 175 & 175 & 0 \\
\hline & Diaphragm Installation & $\mathrm{AG}$ & $\mathrm{AE}, \mathrm{AF}$ & 6 & No & 175 & 198 & 181 & 204 & 46 \\
\hline \multirow[t]{7}{*}{9} & Slab & & & & & & & & & 0 \\
\hline & Reinforcement Work & $\mathrm{AH}$ & $\mathrm{AE}, \mathrm{AF}$ & 46 & Yes & 175 & 175 & 221 & 221 & 0 \\
\hline & S-Form Installation & AI & AG & 17 & No & 181 & 204 & 198 & 221 & 46 \\
\hline & Deck Drain & AJ & AG & 2 & No & 181 & 219 & 183 & 221 & 76 \\
\hline & Concrete Structure & $\mathrm{AK}$ & $\mathrm{AH}, \mathrm{AI}, \mathrm{AJ}$ & 3 & Yes & 221 & 221 & 224 & 224 & 0 \\
\hline & Curing & $\mathrm{AL}$ & $\mathrm{AK}$ & 7 & Yes & 224 & 224 & 231 & 231 & 0 \\
\hline & Moving S-Form & $\mathrm{AM}$ & AL & 2 & No & 231 & 242 & 233 & 244 & 22 \\
\hline \multicolumn{11}{|c|}{ Barrier Structure } \\
\hline & Formwork Installation & $\mathrm{AN}$ & AL & 2,00 & Yes & 231 & 231 & 233 & 233 & 0 \\
\hline & Concrete Structure & $\mathrm{AO}$ & $\mathrm{AN}$ & 3,00 & Yes & 233 & 233 & 236 & 236 & 0 \\
\hline & Curing & $\mathrm{AP}$ & $\mathrm{AO}$ & 7,00 & Yes & 236 & 236 & 243 & 243 & 0 \\
\hline \multicolumn{11}{|c|}{ Drainage } \\
\hline & Excavation $0-2 \mathrm{M}$ & AQ & $\mathrm{AM}$ & 2,00 & No & 233 & 244 & 235 & 246 & 22 \\
\hline & Rc Pipe Installation & $\mathrm{AR}$ & $\mathrm{AQ}$ & 2,00 & No & 235 & 246 & 237 & 288 & 62 \\
\hline Finis & ing & AS & $\mathrm{AP}$ & 5,00 & Yes & 243 & 243 & 248 & 248 & 0 \\
\hline
\end{tabular}


Journal of Engineering and Applied Technology

Vol. 1, No. 2, August 2020, pp. 51-65
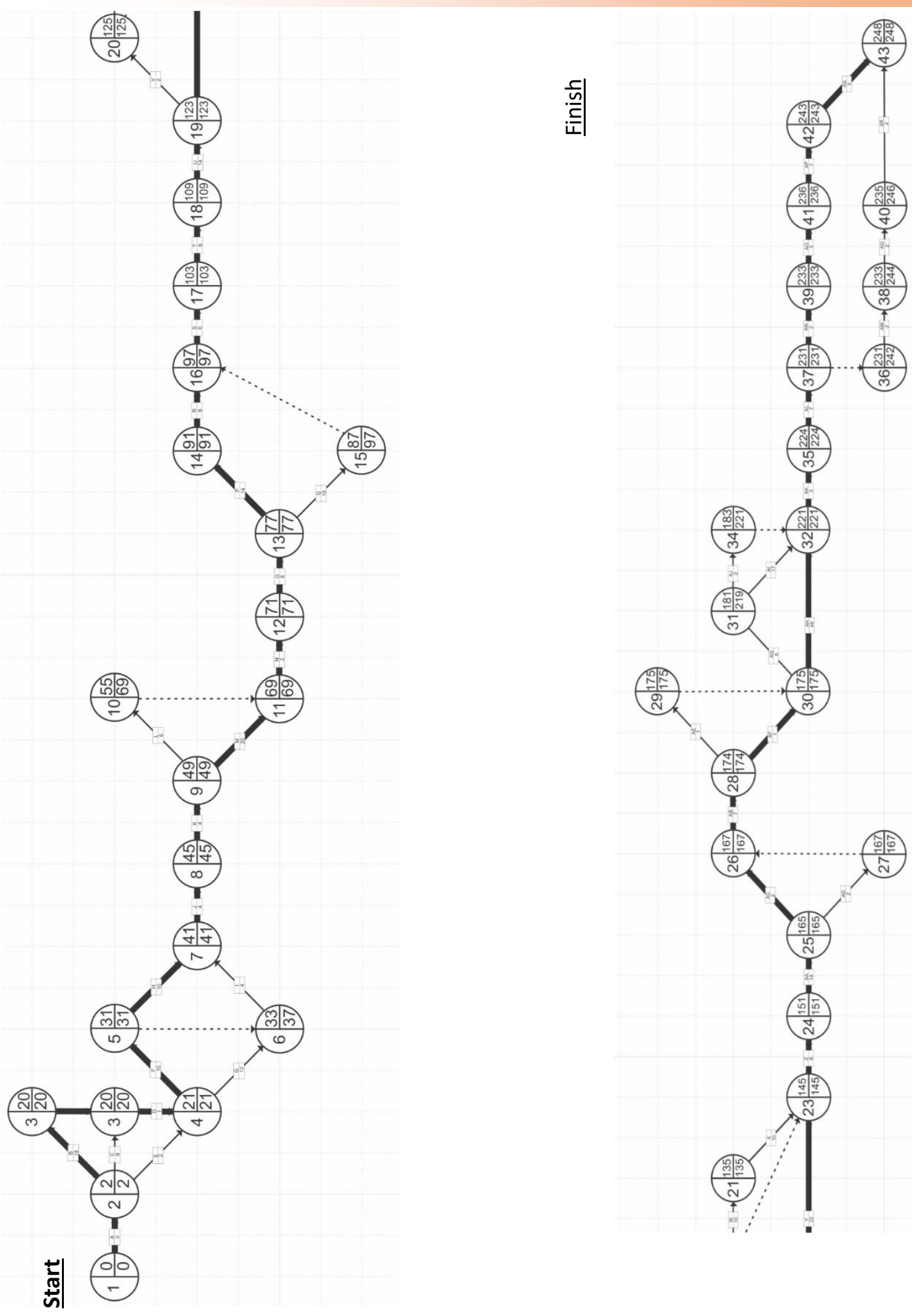

Fig. 4. Network diagram using critical path method (CPM) 
Journal of Engineering and Applied Technology

Vol. 1, No. 2, August 2020, pp. 51-65

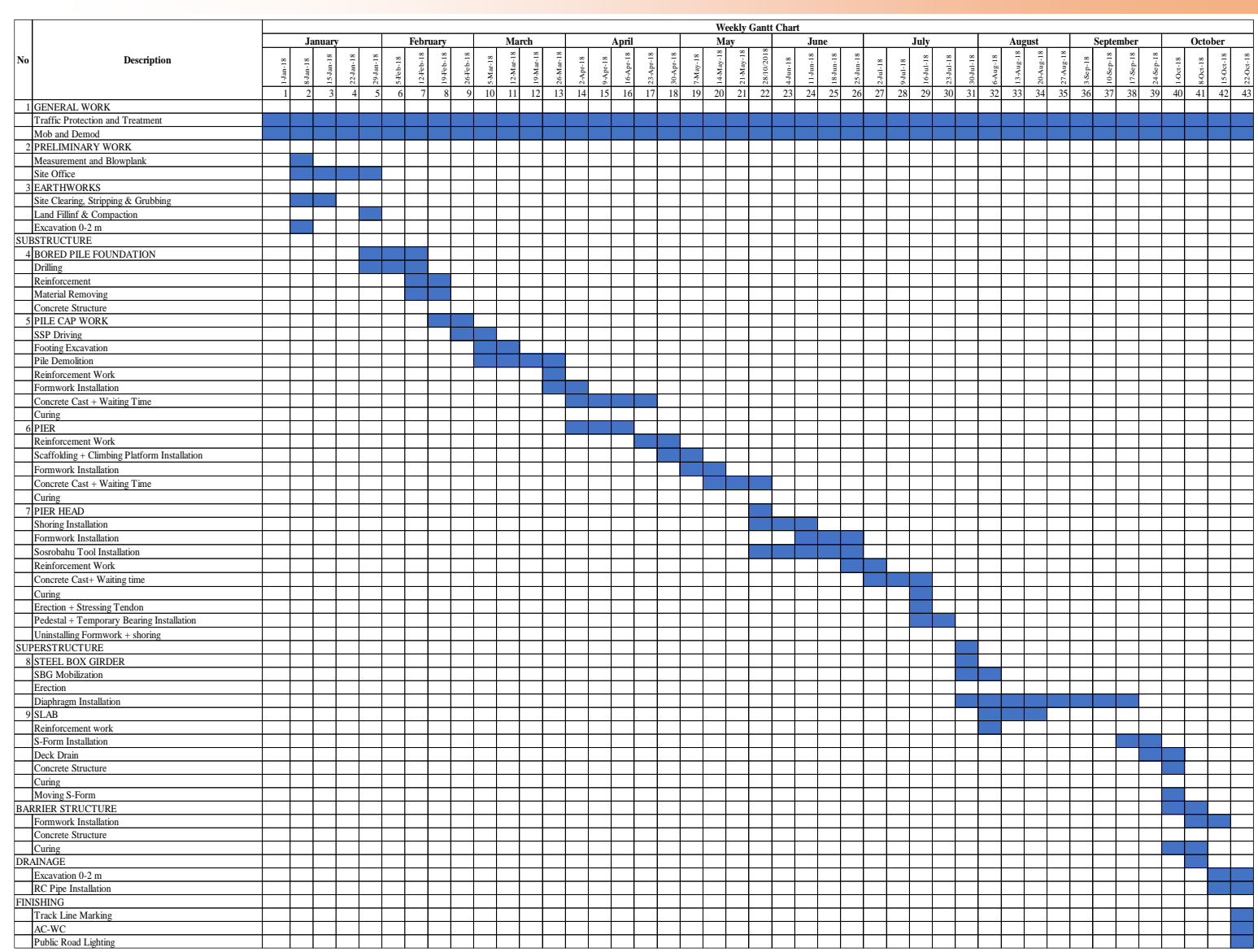

Fig. 5. Gantt chart

General work activities could begin one week before all activities are carried out and become routine activities every day, supporting other activities [13] so that the total project duration will increase by six days. The results of network diagram analysis using the Critical Path Network (CPM) are formed into a Gantt Chart. This Gantt Chart is linked by calendar and weekly blocks (in a week has six working days) [14]. This scheduling concept fundamentally depends on the development methodology, which technically determines the schedule's effectiveness and efficiency. The activity which is the main concern for determining the work method is the pier head section [15]. This section is demanded the optimal duration, but the location of the work in an active toll road area is a serious challenge [16] so that to carry out the development in this section, the Sosrobahu method is chosen, it is aimed at avoiding the direct influence on traffic consumption.

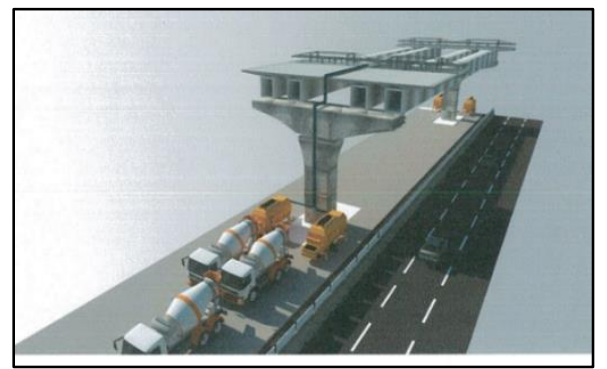

Fig. 6. Project location simulation in the planning of concrete cast for slab structure 


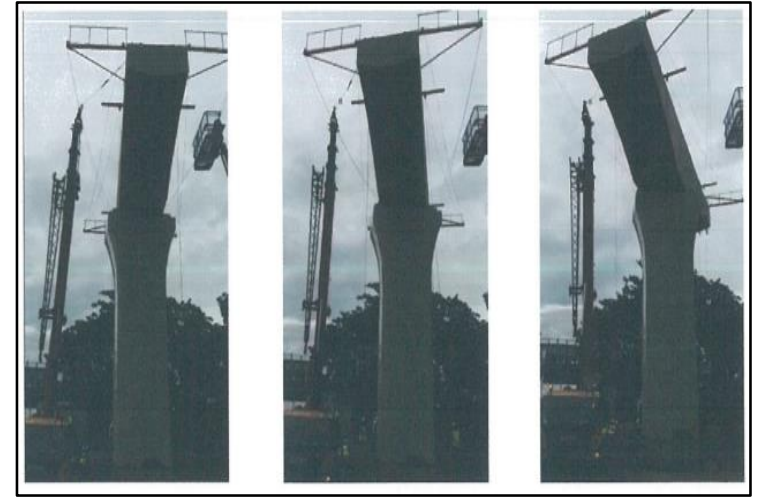

Fig. 7. The momentum of Erection for Pier Head structure

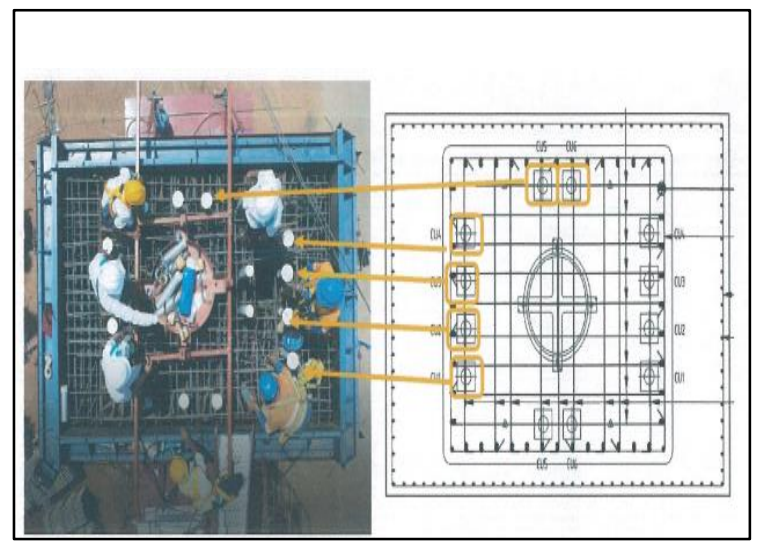

Fig. 9. Installing Sosrobahu template on the top of the crown pier

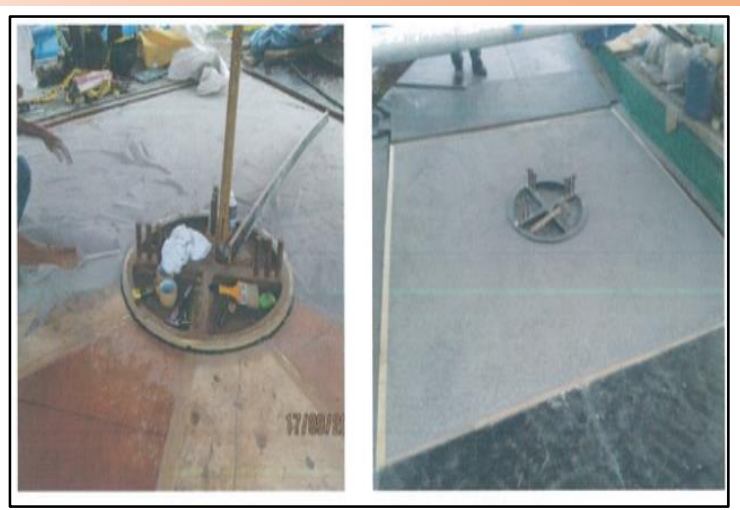

Fig. 8. Installing the first (plywood) and second (white sand) layer dilatation for pierhead

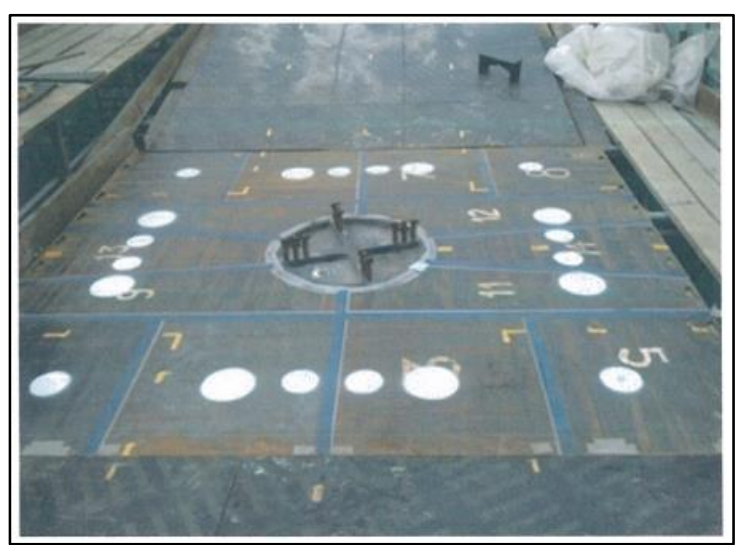

Fig. 10. Installing the third layer dilatation

\section{Conclusion}

The research found that the productivity of equipment and manpower resources affects each other. The optimization of both will provide acceleration and improvement in the quality of work. This productivity data is the fundamental data needed to create an effective and efficient scheduling concept. Through analysis using the Critical Path Method (CPM), the project is known to be planned for completion in 248 working days +6 days $=254$ days, 43 weeks.

\section{References}

[1] S. P. Robbins, Management. Denver: Pearson Prentice Hall, 2007.

[2] R. Wirahadikusumah, Safitri, B. Susanti, and B. Soemardi, "Risk Inclusion in the Reserve Price Estimation for Toll Road Concession Award," J. Traffic Logist. Eng., vol. 2, no. 1, pp. 34-39, 2014, [Online]. Available: http://www.jtle.net.

[3] the Minister of Public Works, Peraturan Menteri Pekerjaan Umum dan Perumahan Rakyat:Pedoman Analisis Harga Satuan Bidang Pekerjaan Umum. 2016. 
Journal of Engineering and Applied Technology

Vol. 1, No. 2, August 2020, pp. 51-65

[4] P. F. Marzuki, H. Permadi, and I. Sunaryo, "Factors Affecting Job Satisfaction of Workers in Indonesian Construction Companies," J. Civ. Eng. Manag., vol. 18, no. 3, pp. 299-309, 2012, DOI: $10.3846 / 13923730.2012 .698889$.

[5] Moder et al., Project Management with CPM, PERT and Precedence Diagraming, 3rd ed. New York: Van Nostrand Reinhold Company, 1983.

[6] P. D. Galloway, "Survey of the construction industry relative to the use of CPM scheduling for construction projects," J. Constr. Eng. Manag., vol. 132, no. 7, pp. 697-711, 2006.

[7] Trihendradic, Microsoft Project 2007 Langkah Cerdas Merencanakan, Menjadwalkan dan Mengontrol Proyek. Yogyakarta: Andi, 2008.

[8] D. Trietsch and K. R. Baker, "PERT 21: Fitting PERT/CPM for use in the 21st century," Int. J. Proj. Manag., vol. 30, pp. 490-502, 2012, [Online]. Available: www.sciencedirect.com.

[9] M. S. Nashwan, "Construction labour productivity: review of factors identified," Int. J. Constr. Manag., pp. 1-13, 2019, DOI: 10.1080/15623599.2019.1627503.

[10] Amaliah, "Penerapan Manajemen Proyek dengan Metode CPM (Critical Path Method) Pada Proyek Pembangunan SPBE," J. Pengabdi. Kpd. Masy., vol. 3, no. May, 2019.

[11] M. Htike and Thein, "Critical path analysis programming method without network diagram," 2018, DOI: doi.org/10.1051/matecconf/201819201027.

[12] A. Freidi, "Determinants of the best practices for successful project management," Int. J. ue- Serv. Sci. Technol., vol. 7, no. 3, pp. 173-186, 2014, DOI: http://dx.doi.org/10.14257/ijunesst.2014.7.3.15.

[13] P. Ivan and S. Andi, "Monitoring and Analysis Project Schedule," 2017.

[14] R. Susanti and L. Lukman, "Studi Eksplorasi Metode Penjadwalan Pada Proyek Konstruksi (Studi Kasus: Proyek Pembangunan Jalan Tol Semarang-Solo Ruas Ruas Ungaran - BAWEN PAKET V," J. Proy. Tek. Sipil, vol. 2, no. 1, pp. 1-3, 2019, DOI: https://doi.org/10.14710/potensi.2019.3607.

[15] M. F. A. Arifin, "Cost analysis for heavy equipment in earthfill work - An optimization of heavy equipment fleet (Case study: Jabung ring dike project)," 2017, DOI: 10.1063/1.4976869.

[16] M. Lu, J. Liu, and W. Ji, "Formalizing a path-float-based approach to determine and interpret total float in project scheduling analysis," Int. J. Constr. Manag., vol. 17, no. 4, pp. 251-263, 2017, DOI: 10.1080/15623599.2016.1207366. 